\title{
Effects of duodenal-jejunal bypass on structure of diaphragm in western diet obese rats ${ }^{1}$
}

Bruna Hart Ulsenheimer', Heloisa Deola Confortim", Léslie Cazetta Jeronimo", Lígia Aline Centenaro"', Ana Tereza Bittencourt Guimarãesıv, Maria Lúcia Bonfleurv, Sandra Lucinei Balbovı, Selma Maria Michelin Matheus ${ }^{\mathrm{VII}}$, Marcia Miranda Torrejais ${ }^{\mathrm{VIII}}$

'Fellow Master degree, Postgraduate Program in Biosciences and Health, Universidade Estadual do Oeste do Paraná (UNIOESTE), Cascavel-PR, Brazil. Scholarship research from CAPES and Araucaria Foundation. Acquisition and interpretation of data, statistical analysis.

"Fellow Master degree, Postgraduate Program in Biosciences and Health, UNIOESTE, Cascavel-PR, Brazil. Technical procedures. I'PhD, Assistant Professor, Center of Medical and Pharmaceutical Sciences, UNIOESTE, Cascavel-PR, Brazil. Conception, design, intellectual and scientific content of the study; manuscript writing.

IVPhD, Assistant Professor, Center for Biological Sciences and Health, UNIOESTE, Cascavel-PR, Brazil. Statistical analysis.

${ }^{\vee}$ PhD, Assistant Professor, Human Physiology Division, Center for Biological Sciences and Health, UNIOESTE, Cascavel-PR, Brazil. Conception, intellectual and scientific content of the study; manuscript writing.

VIPhD, Assistant Professor, Human Physiology Division, Center for Biological Sciences and Health, UNIOESTE, Cascavel-PR. Intellectual and scientific content of the study.

VIIPhD, Assistant Professor, Department of Anatomy, Institute of Biosciences, Universidade Estadual Paulista (UNESP), Botucatu$\mathrm{SP}$, Brazil. Transmission electron microscope analysis.

VIIIPhD, Assistant Professor, Center of Medical and Pharmaceutical Sciences, UNIOESTE, Cascavel-PR, Brazil. Conception, design, intellectual and scientific content of the study; technical procedures.

\section{Abstract}

Purpose: To evaluate the effects of duodenal-jejunal bypass (DJB) on the diaphragm muscle of obese rats fed on a western diet (WD).

Methods: Eighteen male Wistar rats were fed a standard rodent chow diet (CTL group) or WD ad libitum. After 10 weeks, WD rats were submitted to sham (WD SHAM) or duodenal-jejunal bypass (WD DJB). The structure, ultrastructure, collagen content and the morphometry of the neuromuscular junctions (NMJs) were analyzed two months after surgery.

Results: WD SHAM rats displayed an increase in body weight, the Lee index and retroperitoneal and peri-epididymal fat pads compared to the CTL group. DJB did not alter these parameters. The muscle fiber structure and NMJs were similar in the WD SHAM and CTL groups. However, the WD SHAM group showed alterations in the fiber ultrastructure, such as loosely arranged myofibrils and Z line disorganization. In addition, WD SHAM animals presented a considerable amount of lipid droplets and a reduction in the percentage of collagen compared to the CTL group. DJB did not affect the structure or ultrastructure of the muscle fibers or the NMJs in the diaphragm of the WD DJB animals.

Conclusion: Duodenal-jejunal bypass did not improve the alterations observed in the diaphragm of western diet obese-rats.

Key words: Duodenal-jejunal Bypass. Diaphragm. Neuromuscular Junction. Obesity. Muscle Fibers. Rats. 


\section{- Introduction}

Obesity is a public health problem associated with several diseases that directly affect quality of life ${ }^{1}$. Among the associated pathological conditions, those involving the respiratory system stand out - the most common being obstructive sleep apnea and hypoventilation syndrome ${ }^{2}$. The occurrence of respiratory problems in obese people is mainly related to changes in respiratory function ${ }^{3}$. Because of its important function in breathing, the diaphragm must be in continuous rhythmic activity, which requires fatigue resistance from its muscle fibers ${ }^{4}$. However, excessive fat depositsin the chest and abdomen produces compression on the diaphragm, lungs and chest cavity, leading to decreased respiratory system compliance, increased work of breathing, oxygen consumption and respiratory energy expenditure ${ }^{3}$. As a result, the activity of the diaphragm increases in an attempt to maintain adequate alveolar ventilation ${ }^{5}$.

Changes in the respiratory function of obese individuals may be related to alterations in the morphology of the diaphragm ${ }^{5}$. Studies conducted with genetically obese Zucker rats $^{5,6}$ and rats with hypothalamic obesity ${ }^{5}$ reported remodeling and alterations in muscle fiber size ${ }^{5}$ muscle atrophy ${ }^{5,6}$ and fibrosis of the diaphragm ${ }^{6}$. Such alterations to muscle fibers may affect the neuromuscular junctions (NMJs) associated with this muscle, since these two structures are extensively interconnected. Furthermore, Zucker rats have been shown to display alterations to the diaphragm muscle action potential, as manifested by increased height, overshoot and area?

Recently, advances in the treatment of obesity have been achieved with the use of surgical interventions. Bariatric surgery is the most effective treatment for cases of morbid obesity, due to its effectiveness in inducing weight loss and improving comorbidities ${ }^{8}$.
Duodenal-jejunal bypass (DJB) is a type of experimental malabsorptive bariatric surgery, which aims to divert some of the proximal intestine to decrease food absorption9. Studies have shown that in animal models of acquired diabetes or obesity, prior to producing weight loss, this procedure improves glucose homeostasis ${ }^{10-12}$, the lipid profile $^{11}$, attenuates fatty liver disease $\mathrm{e}^{13}$ and prevents atherosclerosis ${ }^{14}$. However, there are no reports on the effects of this type of surgery on the morphology of the diaphragm in obese mice.

The model of animal obesity that most resembles human obesity is that induced by consuming a cafeteria diet ${ }^{15}$. In this diet, animals are offered highly palatable and caloric foods mimicking the westernized diet ${ }^{16}$. The cafeteria diet produces voluntary hyperphagia, rapid weight gain, increased fat mass and generates pre-diabetic parameters such as hyperglycemia and insulin intolerance ${ }^{16,17}$. Thus, our objective was to evaluate the effects of DJB on the structure of muscle fibers and NMJs of the diaphragm in cafeteria diet-induced obese rats.

\section{- Methods}

All experimental procedures were approved by the Ethics Committee on Animal Experiments (CEUA) of the UNIOESTE, under Protocol N ${ }^{\circ} 8709$.

At eight weeks of age, eighteen male Wistar rats (Rattus norvegicus) were randomly divided into two groups: a control group (CTL, $n$ $=6)$ that received a standard diet and water ad libitum, and Western Diet group (WD; $\mathrm{n}=12$ ) that received a cafeteria diet and soda drinks ad libitum. After consuming the cafeteria diet for 10 weeks, the WD group was distributed into two groups: WD sham-operated rats (WD SHAM $n=6$ ); and WD DJB-operated rats (WD DJB; $n=6$ ). For seven days before and seven days after surgery the WD SHAM and WD DJB 
groups were given a liquid cafeteria diet, while the CTL group received the standard liquid diet. Following surgery, both the WD groups continued to receive the cafeteria diet for eight weeks. The animals were kept in cages with standard lighting conditions (06:00 to 18:00) and a controlled temperature $\left(22 \pm 1^{\circ} \mathrm{C}\right)$ throughout the experimental period.

\section{Diets}

The CTL group received the standard rodent diet (Biobase, Brazil) composed of 3.8 $\mathrm{kcal} / \mathrm{g}$ (70\% carbohydrates, $20 \%$ protein and $10 \%$ fat) and water ad libitum. The WD groups received a cafeteria diet. This highly palatable and high calorie diet consisted of standard chow (Biobase, Brazil), Italian salami (Sadia, Brazil), mini bread rolls (Nutrella, Brazil), corn chips (Cheetos, Pepsico, Brazil), marshmallow (Fini, Brazil), mixed sausage (Sadia, Brazil), chocolate cake (Renata, Selmi, Brazil), corn-based cookies (Zadimel, Brazil), mortadella (Frimesa, Brazil), bacon snacks (Trophy, Helena, Brazil), chocolate wafer biscuits (Bauduco, Brazil) and $350 \mathrm{ml}$ of degassed Coca-Cola (Coca-Cola, Brazil) and Guarana (Antarctica, Brazil) per day.

The duodenal-jejunal bypass surgery (DJB) and the sham surgery

The DJB and SHAM surgeries were performed after 10 weeks consuming the cafeteria diet. The perioperative procedures were performed as described by Meguid et al. ${ }^{18}$ and the DJB surgery was performed as described by Jurowich et al. ${ }^{12}$. The animals were fasted for 12 to 16 hours prior to surgery and anesthetized with $1 \%$ isoflurane

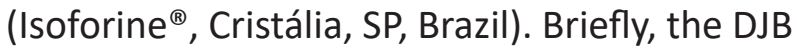
surgery consisted of a laparotomy followed by post-pyloric duodenal transection, closure of the duodenal stump and reconstruction of the intestinal transit through gastrojejunostomy (unionof the pyloricstomach to the jejunum).
To demonstrate the correct execution of the surgery, a saline solution was injected into the pyloric region to test for any constriction or leakage of the liquid. For the sham operation, a midline incision was made into the anterior abdominal wall associated with the movement of the intestinal loops and stomach was performed and then sutured.

\section{Evaluating obesity}

The body weight of the animals was measured from the 8th to 26th weeks of age. At the end of the trial period, the final body weight and nasal-anal length (NAL) were measured to obtain the Lee index - [weight corporal $^{1 / 3}(\mathrm{~g}) /$ nasal-anal length $\left.(\mathrm{cm})\right] \times 1000$, which is considered a parameter for assessing obesity. Then, the animals were desensitized in a $\mathrm{CO}_{2}$ chamber and euthanized by decapitation (guillotine). The retroperitoneal and periepididymal fat was removed and weighed to assess the accumulation of fat.

\section{Collecting the diaphragm}

The animals were placed in a prone position and an incision was made in the midline immediately below the thorax, with the skin and muscle being subsequently folded back. The diaphragm was removed through an incision along its lumbar, costal and sternal portions.

\section{The histological study of the muscle fibers}

The samples of the diaphragm were fixed in Karnovsky's solution and subsequently washed in phosphate buffered saline (PBS) to remove any excess fixative. The samples were embedded in paraplast (SIGMA, Missouri, USA) to facilitate the acquisition of cross sections of the muscle fibers. Subsequently, the muscle fragments were serially sectioned at $7 \mu \mathrm{m}$ using a microtome (R35, Leipzig, China). The obtained 
sections were placed on slides and placed in an oven at $60^{\circ} \mathrm{C}$ for 1 hour. After which the slides were subjected to deparaffinization, hydration and staining with either hematoxylin-eosin for morphological analysis of the muscle fibers or Picrosirius Red, to reveal the collagen. After staining, the slides were dehydrated, cleared and mounted with the aid of Permount (Fisher Scientific ${ }^{\circledR}$, New Jersey, USA).

The ultrastructural analysis of the muscle fibers

Regarding the ultrastructure, samples of the diaphragm muscle were cut into longitudinal fragments (approximately one $\mathrm{mm}$ in width) and immersed in Karnovsky's fixative for mounting. Subsequently, they were washed in $0.1 \mathrm{M}$ phosphate buffer, $\mathrm{pH} 7.3$ (15 minutes) and post-fixed in $1 \%$ osmium tetroxide for two hours. The samples were then washed in distilled water, incubated in $0.5 \%$ uranyl acetate ( 2 hours), dried in acetone and soaked in a mixture of resin and $100 \%$ acetone (12 hours) to form blocks. The desired fields were selected using semi-thin sections $(0.5 \mu \mathrm{m})$ and the ultrathin sections ( $90 \mathrm{~nm}$ thick) were obtained using an ultra-microtome (Ultracut UCT, Leica ${ }^{\circledR}$, Germany). The ultrathin sections were stained with saturated uranyl acetate in $50 \%$ ethyl alcohol (20 minutes) and lead citrate (10 minutes).

The morphological and morphometric study of the neuromuscular junctions

For the analysis of the NMJs, the samples of diaphragm muscle were immersed in Karnovsky's fixative at ambient temperature. They were then sectioned lengthwise into three or four slices using stainless steel blades. The obtained sections were washed in 0.1 $\mathrm{M}$ phosphate buffer, $\mathrm{pH}$ 7.4, for one minute and then subjected to nonspecific esterase reaction ${ }^{19}$ to reveal the cholinesterase enzyme present in the synaptic cleft. The sections were then dehydrated, diaphanized, mounted on slides and covered with cover slips with the aid of Permount (Fisher Scientific ${ }^{\circledR}$, New Jersey, USA).

\section{Analysis of the images}

The muscle fibers were examined under an Olympus microscope coupled to a Bx60 ${ }^{\circledR}$ Olympus DP71 camera (Tokyo, Japan), with the aid of the DP Controller 3.2.1 276 program. Measurement of the muscle fiber area and the quantification of the number of fibers and nuclei were carried out in five, randomly chosen, images (magnification $x 400$ ) per animal. Images of the NMJs were captured in the same microscope described above, with magnification of $\times 200$. The area and larger and smaller diameter sof 50 NMJs were evaluated for each animal. To quantify the collagen, five random images of the samples per animal were captured (magnification x400) using a Leica DMLB ${ }^{\circledR}$ coupled DFC 300 FX camera (Wetzlar, Germany) using QWinV3 software (Leica Microsystems, Wetzlar, Germany). All morphometric analyzes were performed using Image-Pro Plus $6.0^{\circledR}$ software (Media Cybernetics, Maryland, USA). In the ultrastructural analysis, the material was examined and photographed in a transmission electron microscope (CM100, Philips $^{\circledR}$, Netherlands), with 30 regions of muscle fibers being observed per group to quantify the structures in Figure 4J.

\section{Statistical analysis}

Data were initially analyzed using Shapiro-Wilk's normality test. For the analysis of body weight, one-way ANOVA was used together with Tukey's post-test. The Lee index, retroperitoneal and periepididymal fat weight, muscle fiber area and number, the number of peripheral and central nuclei, the percentage of 
collagen, the intrafascicular lipid quantification and the area and largest and smallest diameters of the NMJs were compared using the oneway ANOVA test followed by Bonferroni's post-test and Dunn's Newman-Keuls tests. The ultrastructural analysis, assuming a 1:1 ratio for each group, was evaluated using the Chi2 test for $\mathrm{K}$ proportions, followed by the Marascuilo procedure. The resulting data were expressed as the mean \pm standard deviation or percentage, according to the nature of the variable. In all tests, $\mathrm{P}<0.05$ was considered significant. Analyses were conducted with the aid of the Graph Pad Prism 5.0 ${ }^{\circledR}$ (La Jolla, USA) statistical software.

\section{- Results}

\section{Body parameters}

Before surgery, the body weights of both groups submitted to the cafeteria diet were significantly higher compared to the CTL group $(P=0.0001)$. After the surgery date (10th week of the experimental protocol), the animals in the WD SHAM and WD DJB groups showed little weight loss during the first post-operative week, followed by rapid weight gain compared to the CTL group $(\mathrm{P}=$ 0.0001). At the end of the experiment, the body weight of the animals in the WD SHAM group was significantly higher compared to the CTL group ( $P=0.0001)$, while there was no difference in body weight between the WD SHAM and WD DJB groups (Figure 1A).

The WD SHAM group also presented increases of $4 \%$ in the Lee index $(P<0.05)$, $140 \%$ in the retroperitoneal fat $(P<0.001)$ and $85 \%$ in periepididymal fat $(P<0.01)$ compared to the CTL group. Moreover, regarding these parameters, the WD DJB group presented no significant difference when compared to the WD SHAM group (Figure 1B-D).
Morphological and morphometric analysis of muscle fibers and neuromuscular junctions

The morphology of the diaphragm muscle fibers was found to be similar in appearancein the CTL, WD SHAM and WD DJB groups. The muscle fibers were arranged in fascicles surrounded by perimysium, with the presence of intrafascicular lipids. These fibers were polygonal or rounded in shape with different diameters, peripheral cores, with each fiber surrounded by endomysium.

Analysis of the muscle fiber morphometry showed no significant difference in the area or the number of muscle fibers between the WD SHAM and CTL groups. The animals in the WD DJB group also showed no difference in these variables compared to WD SHAM group (Figure 2A-B). The amount of intrafascicular lipids was similar in the three groups (Figure $2 \mathrm{C}$ ). In addition, there was no difference between the three groups in terms of the number of peripheral and central nuclei (Figure 2D-E). Regarding the percentage of collagen, there was a decrease of $37 \%$ in the WD SHAM group compared to the CTL group $(P<0.05)$. However, there was no significant difference between the WD DJB and WD SHAM groups (Figure 3A-D).

Regarding the ultrastructure of the muscle fibers, the diaphragm of animals form the CTL group had well-defined myofibrils and sarcomeres with organized band $A, I$ and $Z$ line. Peripheral nuclei and lipid droplets were also evident (Figure 4A and $G$ ). In the WD SHAM group (Figure $4 \mathrm{~B}$ and $\mathrm{J}, \mathrm{P}<0.001$ ) more regions were found with loosely arranged myofibrils and $\mathrm{Z}$ line disorganization, compared to the CTL group. Moreover, considerably more lipid droplets were found throughout the intermyofibrillar mitochondria in the WD SHAM group (Figure $4 \mathrm{H}$ ) compared to the CTL group. The WD DJB group (Figure $4 \mathrm{C}$ and I) presented ultrastructural changes similar to 

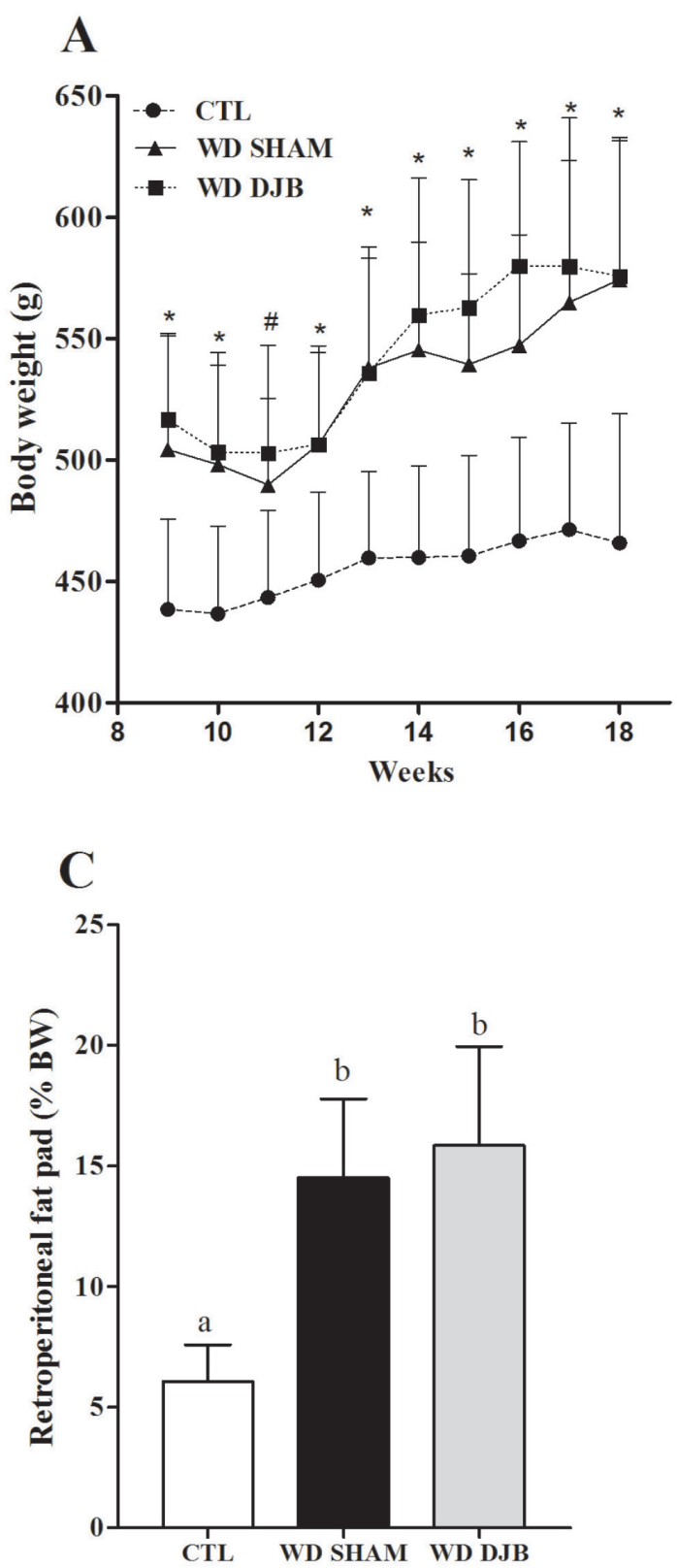
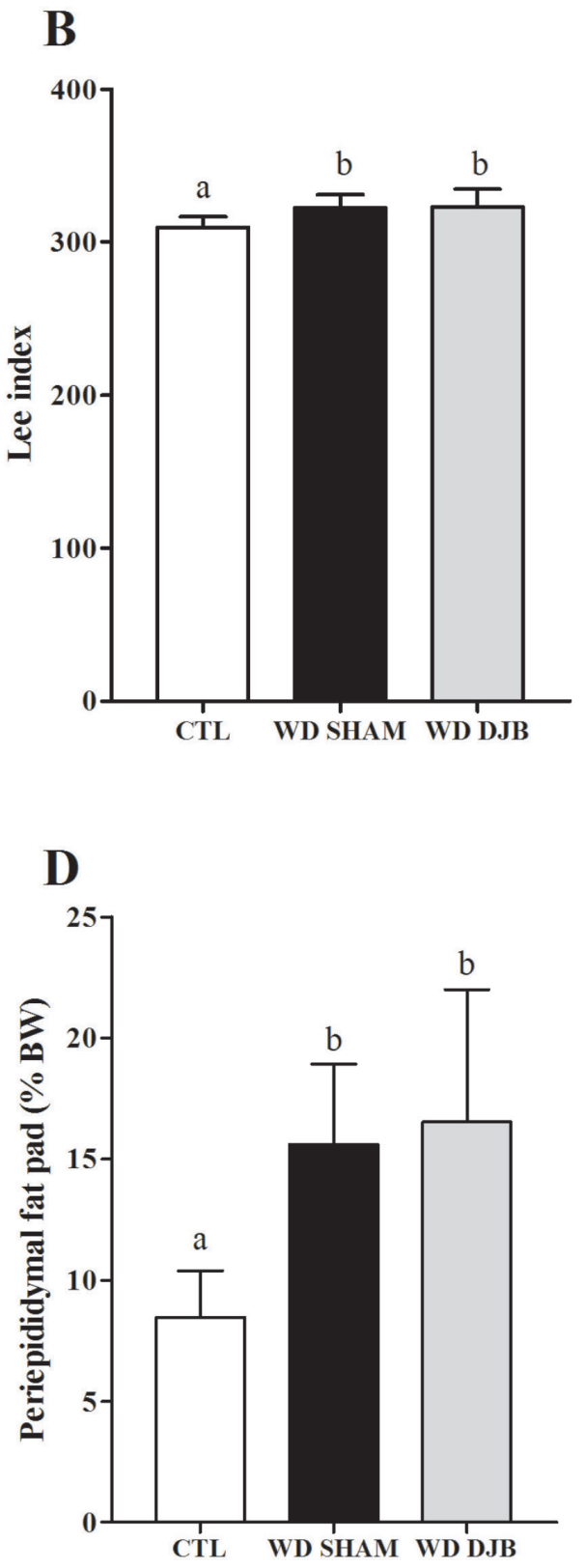

Figure 1 - Body weight (BW) and obesity parameters in CTL and WD rats submitted, or not, to DJB or sham surgeries. (A) Changes in body weight after surgery in the CTL, WDSHAM and WD DJB animals. Values expressed as mean \pm SD from 6-9 rats per group. *WD SHAM and WD DJB vs. CTL, P = 0.0001; \#WD SHAM vs. CTL, $P<$ 0.05 (one-way ANOVA test followed by Tukey's post-test). (B) Lee index and weights of the retroperitoneal (C) and periepididymal (D) fats. Values expressed as mean \pm standard deviation obtained from 8-10 rats per group. The different letters refer to the significant differences between groups, $p<0.05$ (one-way ANOVA followed by Bonferroni's post-test). 
those seen in the WD SHAM group, as there were no significant difference in relation to these changes between the WD DJB and WD SHAM groups (Figure 4J). In the three studied groups, the presence of fragmented nuclei was observed in similar proportions (Figure 4D, E, F and J).

The NMJs observed in the CTL, WD SHAM and WD DJB groups presented varied phenotypes, that is, with round, oval and elliptic shapes (Figure 5A-C). In the morphometric analysis, there was no significant difference in the area and the largest and smallest endplate diameters between the WD SHAM and CTL groups. The WD DJB group also showed no significant difference in the size of the NMJs compared to WD SHAM group (Figure 5D-F).

\section{- Discussion}

In this study, we observed that, in the short-term, DJB failed to reverse the body parameters, the reduction of collagen and ultrastructural changes in the muscle fibers caused by obesity in cafeteria diet-induced obese rats. However, the DJB caused no changes in the morphology or morphometry in the muscle fibers of the diaphragm.

In this study, the cafeteria diet given to the animals induced obesity, since it led to body weight gain and increased stocks of retroperitoneal and periepididymal fat. The increase in the Lee index, analogous to the human body mass index, also confirmed the obesity of the animals. These data are consistent with those of other studies that reported increases in these body parameters in rats fed with the cafeteria diet ${ }^{20}$. In order to investigate anypossible beneficial effects of DJB surgery, the animals submitted to the cafeteria diet continued to receive the same diet after the surgery. DJB surgery is a bariatric procedure capable of improving glucose homeostasis in animal models of acquired diabetes and diet- induced obesity ${ }^{13,20}$, regardless of body weight loss. In this study, there were no changes in body weight, the weight of retroperitoneal and periepididymal fator the Lee index eight weeks after the DJB. These results are in agreement with a previous study that also showed no reduction in these parameters in obese animals that continued to receive a cafeteria diet for eight weeks post-DJB ${ }^{20}$. In the study by $\mathrm{Hu}$ et al. ${ }^{11}$, DJB was also unable to reduce the body weight of animals previously fed with a highcalorie diet and a standard diet for 12 weeks after surgery. According to Patel et al. ${ }^{21}$, rats submitted to DJB showed no changes in the concentration of fasting ghrelin, a hormone involved in regulating food intake. Thus, it is suggested that animals did not lose weight after DJB because this surgical procedure does not provoke a change in food intake. Furthermore, one study reports ${ }^{22}$ intestinal adaptation in obese rats after DJB, which suggests an increase in intestinal absorption.

Although the cafeteria diet led to increased stocks of retroperitoneal and periepididymal fat, the diaphragm muscle of animals in the WD SHAM group showed no increase in intrafascicular lipids compared to the CTL group. DJB surgery did not alter the amount of lipids in that muscle. Goodpaster et al. ${ }^{23}$ reported an increase in the amount of intramuscular lipids in the vastus lateralis muscle of obese individuals and Gray et al. ${ }^{24}$ noted a reduction in the amount of intramuscular lipids of obese people after weight loss induced by Roux-Y gastric bypass. The diaphragm may be less susceptible to such changes compared to the other skeletal muscles, possibly due to its constant activation during respiratory functions.

It is known that cafeteria diet-induced obesity tends to reduce the muscle fiber area of the hind limb muscles ${ }^{25}$. However, the cafeteria diet and DJB surgery did not alter the structure of the diaphragm, as no changes were observed in the area, number of muscle fibers or the number of peripheral or central nuclei. Gosselin et al. ${ }^{26}$ also noted that the size of the muscle fibers in 
A

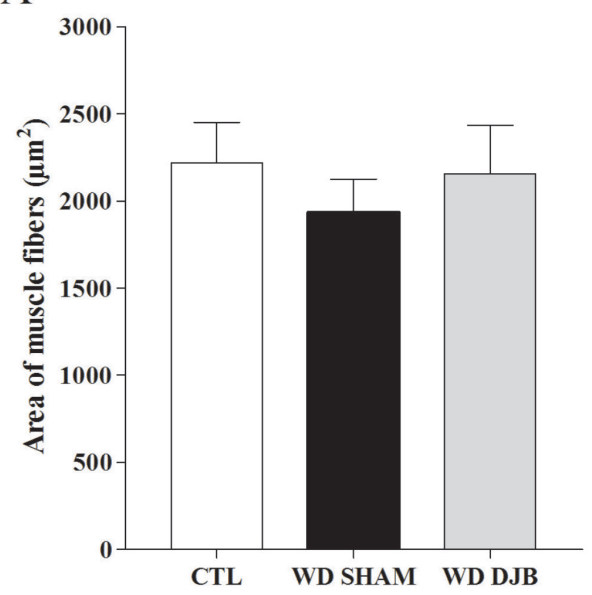

$\mathrm{C}$

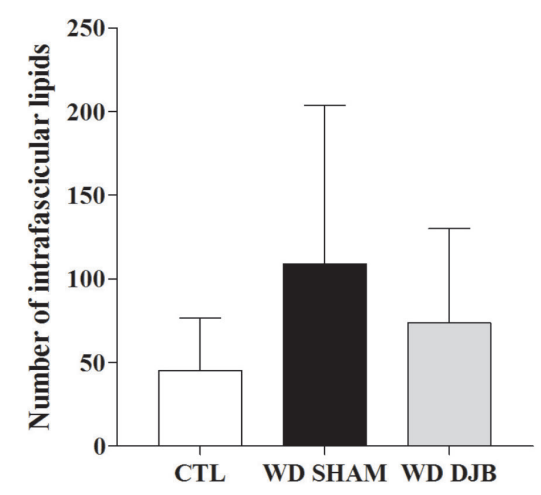

$\mathrm{E}$

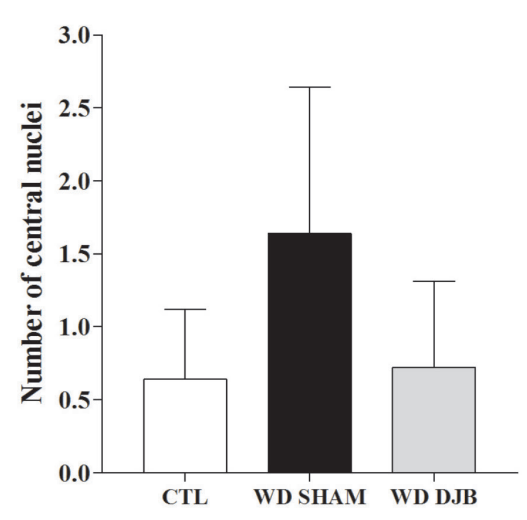

$\mathrm{B}$

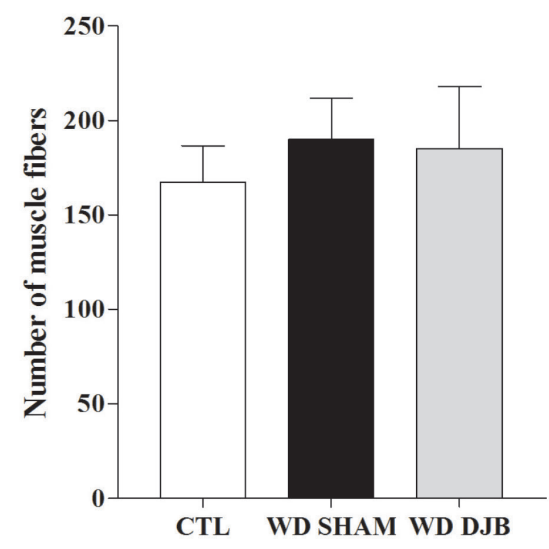

$\mathrm{D}$

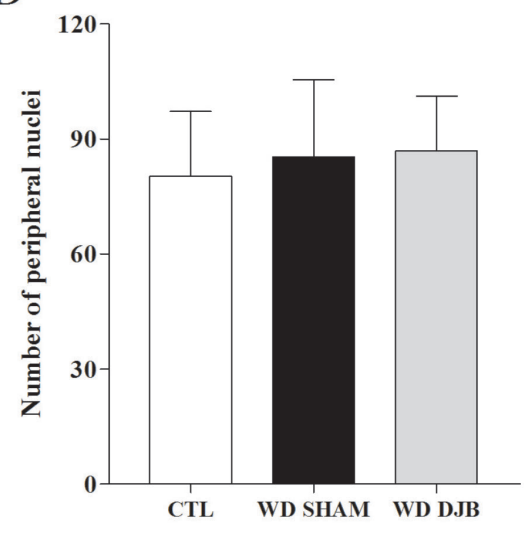

Figure 2 - Morphometric analysis of the diaphragm muscle of the animals in the CTL, WD SHAM and WD DJB groups. (A) Muscle fiber area. (B) Number of muscle fibers. (C) Number of intrafascicular lipids. (D) Number of peripheral nuclei. (E) Number of central nuclei. Values expressed as mean \pm standard deviation obtained from 5 rats per group. (Number of peripheral nuclei: Kruscal-Wallis Test followed by Dunn's post-test; Further analysis: one-way ANOVA test followed by the Newman-Keuls post-test). 

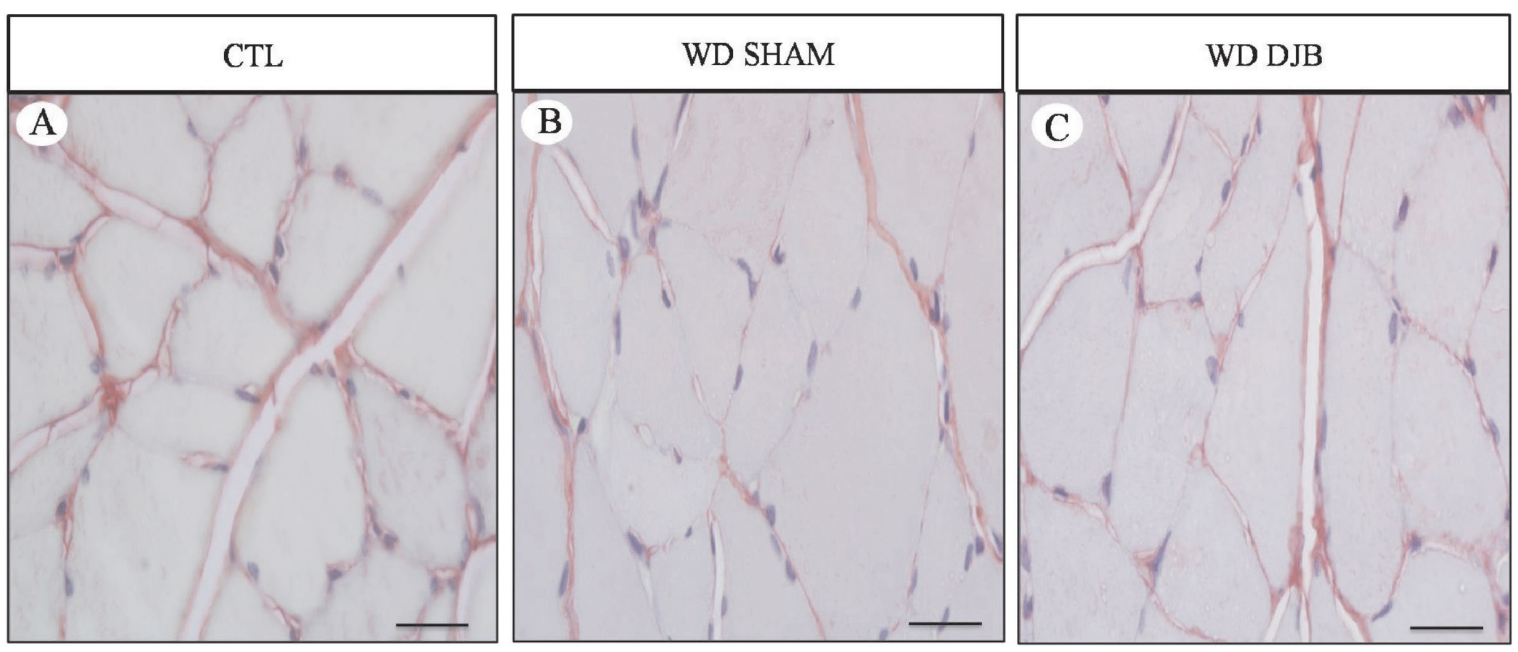

D

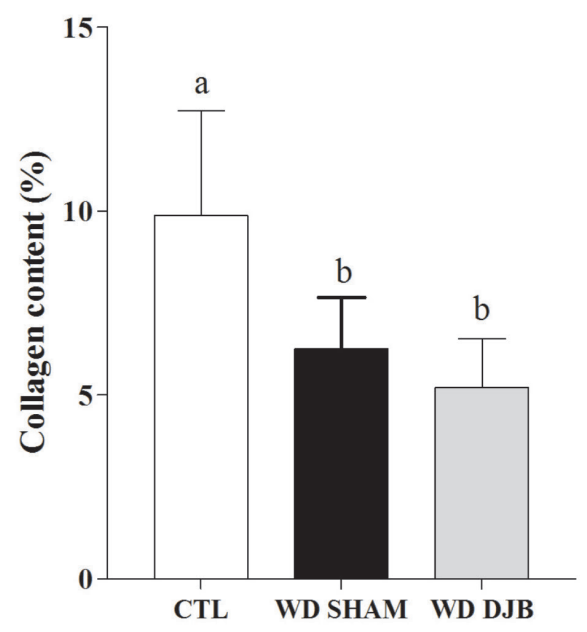

Figure 3 - Photomicrographs of the collagen present in the muscle fibers of the diaphragm in animals from the CTL (A), WD SHAM (B) and WD DJB (C) groups. Cross section. Picrosirius red without polarized light. Bar = $20 \mu \mathrm{m}$. (D) Percentage of collagen in the diaphragm. Values expressed as mean \pm standard deviation obtained from 5 rats per group. a represents a significant difference $P<0.05$. (One-way ANOVA followed by NewmanKeuls post-test).

the diaphragm was unaltered in young and senescent rats, even after physical training. Again, this suggests that the constant activity of the diaphragm muscles in maintaining the respiratory function becomes the resilient to the process of muscle atrophy.

Regarding the ultrastructure of the diaphragm, more regions were found to have foci of loosely arranged myofibrils and disorganized $Z$ lines in the animals from the WD SHAM group compared to the CTL group and DJB did not reverse these changes. It is suggested that these changes in the myofibrils may jeopardize the functioning of the muscle ${ }^{27}$, causing losses in the diaphragmatic dynamics. In addition, the presence of fragmented nuclei was observed in the three groups. Fragmentation of the nucleus is seen 


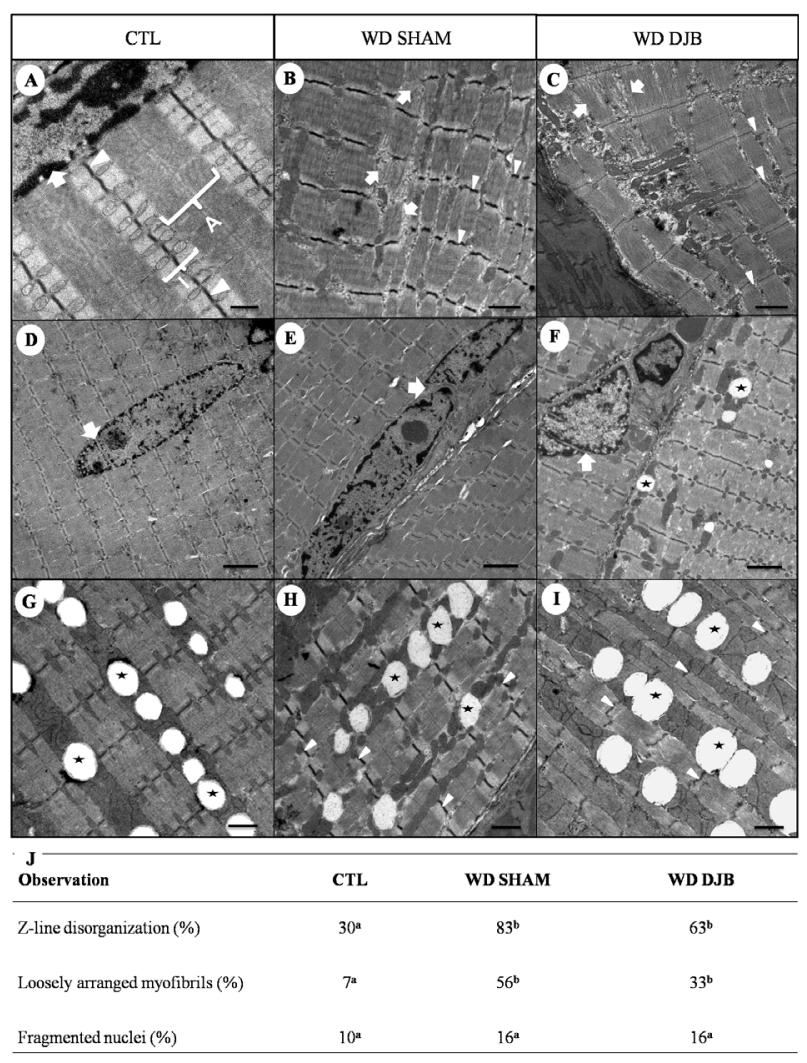

Figure 4 - Transmission electron micrographs of the diaphragm muscle from rats. Longitudinal section. The right column corresponds to the CTL group; the central column corresponds to the WD SHAM group; the left column corresponds to the WD DJB group. (A) Note the peripheral nucleus (short arrow), organized $Z$ line (arrowhead), Band A (A) and Band I (I). Bar = $500 \mathrm{~nm}$. (B, C) Note the disorganization of the $Z$ line (arrowhead), foci of loosely arranged myofibrils (short arrow). Bar $=1$ $\mu \mathrm{m}$. (D, E and F) Note the presence of fragmented nuclei (short arrow) and lipid droplets (star). Bar = $2 \mu \mathrm{m}$. (G, H and I) lipid droplets are visible (star), and $Z$ line disorganization (arrowhead). Bar $=1 \mu \mathrm{m}$. (J) Ultrastructural changes observed in the animals from the CTL, WD SHAM and WD DJB groups. Values expressed as percentages obtained from 2 to 3 rats per group. Different letters in the same line represent statistically significant differences, $P<0.001$. ( $x 2$ test followed by the Marascuilo monitoring test). in the process of apoptosis, which occurs in cases of cellular renewal and defense against diseases $^{28}$.

To the best of our knowledge, this is the first study to investigate the association between cafeteria diet-induced obesity and the percentage of collagen in the diaphragm. A decrease in the percentage of collagen was observed in the WD SHAM group compared to the CTL group. This reduction in collagen may be related to its decreased synthesis and/or increased degradation. According to $\mathrm{Hu}$ et al. ${ }^{11}$, moderately obese rats submitted to a high calorie diet have a high concentration of leptin. This hormone enhances the activity of the metalloproteinases MMP-22929 and the mRNA expression of MMP- $9^{30}$, enzymes responsible for collagen degradation. As the percentage of collagen in the diaphragm muscle was similar in the WD DJB and WD SHAM groups, it is suggested that the continued use of the cafeteria diet and the maintenance of post-DJB body weight maintained the leptin concentration and activity of matrix metalloproteinases high, leading to increased degradation of collagen in relation to its synthesis.

Again, to the best of our knowledge, this is the first time the effect of the cafeteria diet and DJB on NMJ morphometry has been investigated. When compared to CTL group, the cafeteria diet did not alter the morphology of the NMJs in the WD SHAM group. Moreover, DJB surgery did not modify the size of these structures compared to the WD SHAM group. However, we found that the WD DJB group showed increases of $29 \%$ in the area, $28 \%$ in largest and $16 \%$ in the smallest diameter of the NMJs compared to the CTL group. This increase in the size of the NMJs may be related to a possible remodeling of the muscle fibers. Due to the lack of studies involving the characterization of these structures in obesity and after bariatric surgery, more studies are needed to clarify these findings. 

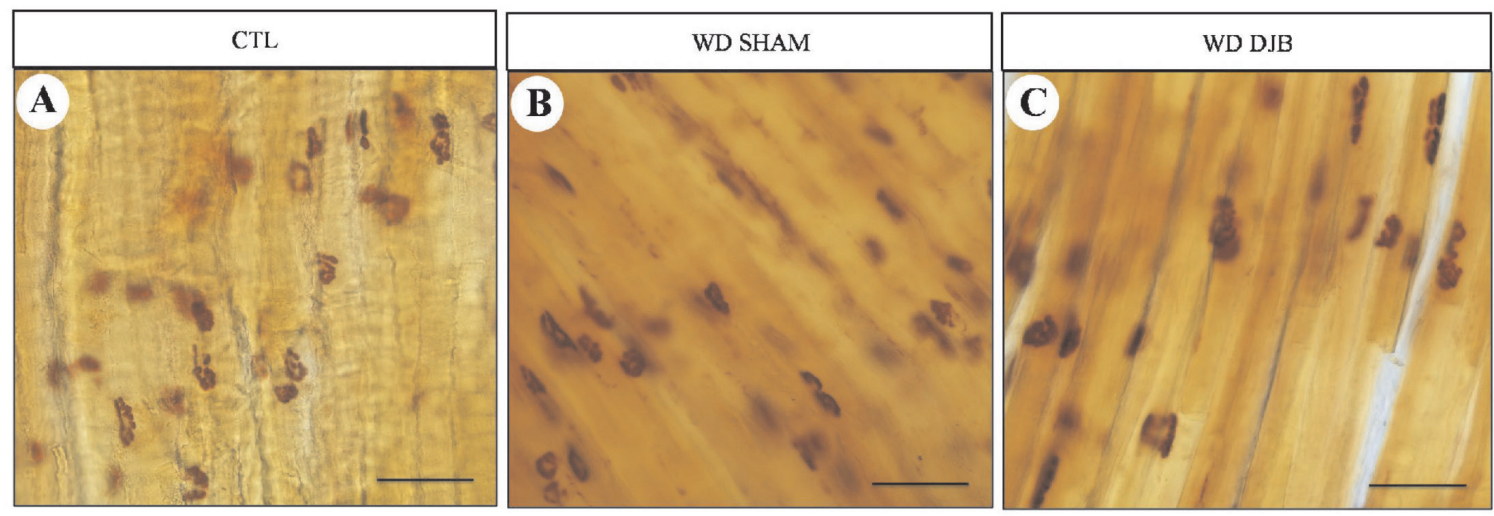

D

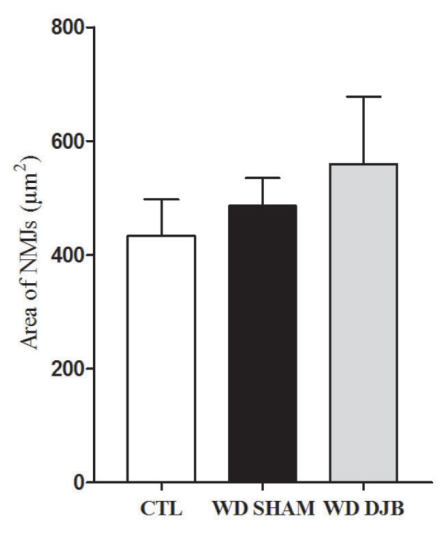

E

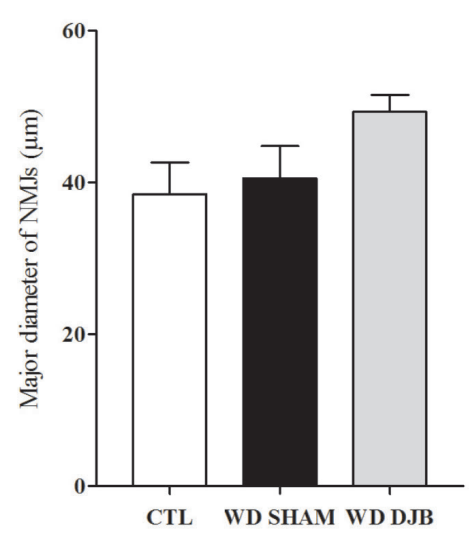

F

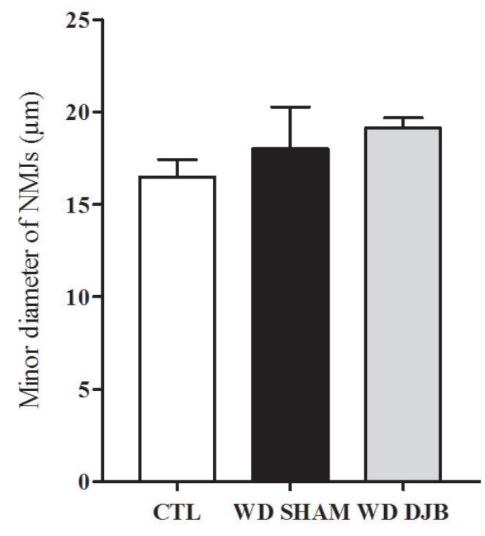

Figure 5 - Photomicrographs of the NMJs from the diaphragm of rats in the CTL (A), WD SHAM (B) and WD DJB (C) groups. Longitudinal section. Nonspecific esterase. (D) NMJ area (E) largest NMJ diameter. (F) Smallest NMJ diameter. Bar $=100 \mu \mathrm{m}$. Values expressed as mean \pm standard deviation obtained from 5 rats per group. (One-way ANOVA followed by the Newman-Keuls post-test).

\section{- Conclusions}

Obesity induced by the cafeteria diet caused ultrastructural changes in the muscle fibers of the diaphragm and reduced the percentage of collagen in that muscle. Duodenaljejunal bypass surgery did not reduce body weight or the weight of the fat in obese animals and did not reverse the deleterious effects on the diaphragm.

\section{- References}

1. Stein CJ, Colditz GA. The epidemic of obesity. J Clin Endocrinol Metab. 2004
Jun;89(6):2522-5. PMID: 15181019.

2. Murugan AT, Sharma G. Obesity and respiratory diseases. Chron Respir Dis. 2008;5(4):233-42. PMID: 19029235.

3. Wei YF, Wu HD. Candidates for bariatric surgery: morbidly obese patients with pulmonary dysfunction. J Obes. 2012;2012:1-6. PMID: 22685636.

4. Polla B, D’Antona G, Bottinelli R, Reggiani C. Respiratory muscle fibres: specialisation and plasticity. Thorax. 2004 Sep;59(9):80817. PMID: 15333861.

5. Scano G, Stendardi L, Bruni GI. The respiratory muscles in eucapnic obesity: their role in dyspnea. Respir Med. 2009 
Sep;103(9):1276-85. PMID: 19450957.

6. Allwood MA, Foster AJ, Arkell AM, Beaudoin MS, Snook LA, Romanova N, Murrant CL, Holloway GP, Wright DC, Simpson JA. Respiratory muscle weakness in the Zucker diabetic fatty rat. Am J Physiol Regul Integr Comp Physiol. 2015 Oct;309(7):780-7. PMID: 26246509.

7. van Lunteren E, Moyer M. Altered diaphragm muscle action potentials in Zucker diabetic fatty (ZDF) rats. Respir Physiol Neurobiol. 2006 Sep;153(2):157-65. PMID: 16311078.

8. Pories WJ. Bariatric surgery: risks and rewards. J Clin Endocrinol Metab. 2008 Nov;93:89-96. PMID: 18987275.

9. Runkel N, Colombo-Benkmann $M$, Hüttl TP, Tigges $H$, Mann $O$, Sauerland $S$. Bariatric surgery. Dtsch Arztebl Int. 2011 May;108(20):341-6. PMID: 21655459.

10. Breen DM, Rasmussen BA, Kokorovic A, Wang R, Cheung GW, Lam TK. Jejunal nutrient sensing is required for duodenaljejunal bypass surgery to rapidly lower glucose concentrations in uncontrolled diabetes. Nat Med. 2012 Jun;18(6):950-5. PMID: 22610279.

11. Hu C, Zhang G, Sun D, Han H, Hu S. Duodenal-jejunal bypass improves glucose metabolism and adipokine expression independently of weight loss in a diabetic rat model. Obes Surg. 2013 Sep;23(9):143644. PMID: 23636998.

12. Jurowich CF, Rikkala PR, Thalheimer A, Wichelmann C, Seyfried F, Sander V, KreissI M, Germer CT, Koepsell H, Otto C. Duodenal-jejunal bypass improves glycemia and decreases SGLT1-mediated glucose absorption in rats with streptozotocininduced type 2 diabetes. Ann Surg. 2013 Jul;258(1):89-97. PMID: 23478528.

13. Ebertz CE, Bonfleur ML, Bertasso IM, Mendes MC, Lubaczeuski C, Araujo AC, Paes AM, Amorim EMP, Balbo SL. Duodenal jejunal bypass attenuates non-alcoholic fatty liver disease in western diet-obese rats. Acta Cir Bras. 2014 Sep;29(9):609-14. PMID: 25252208.

14. Chen X, Huang Z, Ran W, Liao G, Zha L, Wang $Z$. Type 2 diabetes mellitus control and atherosclerosis prevention in a nonobese rat model using duodenal-jejunal bypass. Exp Ther Med. 2014 Sep;8(3):85662. PMID: 25120614.

15. Cesaretti MLR, Koblmann-Junior $O$. Experimental models of insulin resistance and obesity: lessons learned. Arc Bras Endocrinol Metab. 2006;50(2):190-7. PMID: 16767285.

16. Sampey BP, Vanhoose AM, Winfield HM, Freemerman AJ, Muehlbauer MJ, Fueger PT, Newgard CB, Makowski L. Cafeteria diet is a robust model of human metabolic syndrome with liver and adipose inflammation: comparison to high-fat diet. Obesity. 2011 Jun;19(6):1109-17. PMID: 21331068.

17. Castell-Auví $A$, Cedó $L$, Pallarès V, Blay $M$, Ardévol A, Pinent M. The effects of a cafeteria diet on insulin production and clearance in rats. Br J Nutr. 2012 Oct;108(7):1155-62. PMID: 22152054.

18. Meguid MM, Ramos EJ, Suzuki S, Xu Y, George ZM, Das UN, Hughes K, Quinn $R$, Chen C, Marx W, Cunningham PR. A surgical rat model of human Roux-en-Y gastric bypass. J Gastrointest Surg. 2004 JulAug;8(5):621-30. PMID: 15240001.

19. Lehrer GM, Ornstein L. A diazo coupling method for the electron microscopic localization of cholinesterase. Biophys Biochem Cytol. 1959 Dec;6:399-406. PMID: 14415404.

20. Araujo A, Bonfleur ML, Balbo S, Ribeiro R, Freitas A. Duodenal-jejunal bypass surgery enhances glucose tolerance and betacell function in Western diet obese rats. Obes Surg. 2012 May;22(5):819-26. PMID: 22411572.

21. Patel RT, Shukla AP, Ahn SM, Moreira M, Rubino F. Surgical control of obesity and diabetes: the role of intestinal vs. gastric mechanisms in the regulation of body weight and glucose homeostasis. Obesity. 2014 Jan;22(1):159-69. PMID: 23512969.

22. Li B, LuY, Srikant CB, GaoZH, Liu1 JL. Intestinal adaptation and Reg gene expression induced by antidiabetic duodenal-jejunal bypass surgery in Zucker fatty rats. Am J Physiol Gastrointest Liver Physiol. 2013 
Apr;304(7):635-45. PMID: 23370676.

23. Goodpaster BH, Theriault R, Watkins SC, Kelley DE. Intramuscular lipid content is increased in obesity and decreased by weight loss. Metabolism. 2000 Apr;49(4):467-72. PMID: 10778870.

24. Gray RE, Tanner CJ, Pories WJ, MacDonald KG, Houmard JA. Effect of weight loss on muscle lipid content in morbidly obese subjects. Am J Physiol Endocrinol Metab. 2003 Apr;284(4):726-32. PMID: 12488242.

25. Sishi B, Loos B, Ellis B, Smith W, Toit EF, Engelbrecht AM. Diet-induced obesity alters signalling pathways and induces atrophy and apoptosis in skeletal muscle in a prediabetic rat model. Exp Physiol. 2011 Feb;96(2):17993. PMID: 20952489.

26. Gosselin LE, Betlach M, Vailas AC, Thomas DP. Training-induced alterations in young and senescent rat diaphragm muscle. J Appl Physiol. 1992 Apr;72(4):1506-11. PMID: 1592743.

27. Faulkner JA, Brooks SV, Opiteck JA. Injury to skeletal muscle fibers during contractions: conditions of occurrence and prevention. Phys Ther. 1993 Dec;73(12):911-21. PMID:

\section{9.}

28. Ziegler U, Groscurth P. Morphological features of cell death. News Physiol Sci. 2004 Jun;19:124-8. PMID: 15143207.

29. Schram K, De Girolamo S, Madani S, Munoz $D$, Thong F, Sweeney $G$. Leptin regulates MMP-2, TIMP-1 and collagen synthesis via p38 MAPK in HL-1 murine cardiomyocytes. Cell Mol Biol Lett. 2010 Dec;15(4):551-63. PMID: 20683677.

30. Tao M, Yu P, Nguyen BT, Mizrahi B, Savion $\mathrm{N}$, Kolodgie FD, Virmani R, Hao S, Ozaki CK, Schneiderman J. Locally applied leptin induces regional aortic wall degeneration preceding aneurysm formation in apolipoprotein E-deficient mice. Arterioscler Thromb Vasc Biol. 2013 Feb;33(2):311-20. PMID: 23220275.

\section{- Acknowledgements}

To Assis Roberto Escher for animal care, and UNESP-Botucatu, especially Gelson Rodrigues of Anatomy Department by the technical support.

\section{Correspondence:}

Márcia Miranda Torrejais

85.819-110 Cascavel - PR Brasil

Tel./Fax: (55 45)3220-3194

mmtorrejais@yahoo.com.br

Conflict of interest: none

Financial sources: Araucaria Foundation and CAPES
Received: Sep 15, 2016

Review: Nov 17, 2016

Accepted: Dez 18, 2016

${ }^{1}$ Research performed at Laboratory of Experimental Morphology, Center for Biological Sciences and Health, Universidade Estadual do Oeste do Paraná (UNIOESTE), Cascavel-PR, Brazil. Part of Master degree thesis, Postgraduate Program in Biosciences and Health. Tutor: Márcia Miranda Torrejais. 